J. Chosun Natural Sci.

Vol. 7, No. 2 (2014) pp. $75-78$

http://dx.doi.org/10.13160/ricns.2014.7.2.75

Review Areticle

\title{
A Short Review on the Application of Combining Molecular Docking and Molecular Dynamics Simulations in Field of Drug Discovery
}

\author{
Gugan Kothandan $^{1 \dagger}$ and Jagadeesan Ganapathy ${ }^{2}$
}

\begin{abstract}
Computer-aided drug design uses computational chemistry to discover, enhance, or study drugs and related biologically active molecules. It is now proved to be effective in reducing costs and speeding up drug discovery. In this short review, we discussed on the importance of combining molecular docking and molecular dynamics simulation methodologies. We also reviewed the importance of protein flexibility, refinement of docked complexes using molecular dynamics and the use of free energy calculations for the calculation of accurate binding energies has been reviewed.
\end{abstract}

Key words: Structure-based Drug Design, Docking, Drug Design, Molecular Dynamics

\section{Introduction}

The development of new drugs is difficult and it is one of the most challenging tasks of modern day's science. The combined efforts of the pharmaceutical industry, biotech companies, regulatory authorities, academic researchers, and other private and public sectors led to the development of new and more effective drugs and also contributed to the advance of science. The approaches and methodologies used in drug design have changed over time. Today, the field of drug development looks highly productive with the finding of new targets, the usage of rational combinatorial chemistry for the production of libraries of compounds, the generation of genetically modified animal models for the development and testing of new drugs, and the possibility of using ultra-high-throughput test techniques for the screening of large libraries. However, despite all these advances, the revolutionary era of drug design has not arrived yet ${ }^{[1-3]}$. There is no unique solution to a drug design problem. The appropriate experimental techniques or computational methods to use will depend on the characteristics of the system itself and the information available.

\footnotetext{
Centre of Advanced Study in Crystallography and Biophysics, University of Madras, Guindy Campus, Chennai-600025, India

${ }^{2}$ Department of Physics, Presidency College, Chennai 600005, India

Corresponding author: guganmsc@gmail.com

(Received: May 27, 2014, Revised : June 13, 2014,

Accepted : June 25, 2014)
}

Structure-based drug design (SBDD) has become an established pattern in modern drug discovery to search for novel drug-like molecules able to bind to biomolecules of important pharmaceutical interest ${ }^{[4]}$. Because it is highly expensive and highly time consuming to prepare and assay large numbers of small molecules, there is strong interest in computational methods that can accurately predict small molecule binding affinities and hence reduce the time and resources. The low costs and speed at which docking studies can be conducted make the procedure valuable to prioritize compounds to assay in a drug discovery program, or to assist the design of improved ligands. Various computational chemistry techniques are often used to assist the structure based drug design process ${ }^{[5]}$.

However, there is no unique solution to a drug design problem. The appropriate experimental techniques or computational methods to use will depend on the characteristics of the system itself and the information available. A variety of computational approaches can be applied at different stages of the drug-design process from lead identification to lead-optimization. The main focus is on decreasing experimental costs and reducing times and it was tried many a times and only few successful applications have been reported ${ }^{[6-8]}$. The lack of success has led to opt for experimental solutions and other researchers focused their attention on the improvement of computational protocols such as incorporation of protein flexibility in the docking process, extensive 
exploration of the ligand conformation within the binding site, refinement and stability evaluation of the final complexes, and estimation of the binding free energies. This prompted us to write a short review on the improvement of computational protocols.

We are actively reporting short reviews covering different in silico applications such as Pseudoreceptors, Molecular Mechanics/Poisson-Boltzmann Surface Area (MM-PBSA) method in Drug Discovery, development of search algorithm in docking and importance of partial charges ${ }^{[9-13]}$. In this review, we report on the importance of protein flexibility, refinement of docked complexes using molecular dynamics and the use of free energy calculations for the calculation of accurate binding energies.

\section{Molecular Docking and Molecular Dynamics Simulation}

Docking is a method which predicts the preferred orientation of one molecule (ligand or receptor) to a second (ligand or receptor) when bound to each other to form a stable complex ${ }^{[14]}$. The process of binding a small molecule to its protein target is not simple and several factors need to be considered. DOCK, AutoDock, FlexX, GOLD, and GLIDE are some of the popular docking software's. Similarly, Molecular dynamics simulations are one of the widely used computational techniques for studying the behavior of biological macromolecules ${ }^{[15]}$. They are really helpful in understanding the dynamic behavior of protein and small molecules. Amber, CHARMM, GROMOS and NAMD are some of the programs for MD simulations. Both the above techniques can be combined to predict more reliable enzyme complexes. Both these techniques have their advantages and disadvantages. Therefore, combination of both these techniques is helpful in improving the drug design process.

\section{Importance of Protein Flexibility in Docking}

It has been widely accepted that the lock and key interaction of a receptor and its ligand is not an accurate description of most biological complexes. The ligandprotein interactions consider both of them are flexible and complement each other. Receptor conformation is important for protein flexibility and many studies have highlighted the importance of conformation of receptor for docking studies ${ }^{[16]}$. The three dimensional (3-D) structure of both ligand and protein are necessary for the application of docking techniques. Some of the proteins do not have an experimentally determined structure and in those cases a 3D structure can be predicted. It is of great importance to carefully prepare the structure of the receptor before docking. It is always desirable to treat the receptor as flexible molecule to avoid bias and to allow the conformational changes during docking. Different approximate methods are used to consider receptor flexibility.

The main advantage of considering receptor flexibility is it will reduce the risks associated with inadequate conformation of the protein target during the docking process $^{[17]}$. Recent advances in docking algorithms have allowed incorporation of ligand flexibility and, to less extent, protein mobility, during the docking procedure. Most modern algorithms account for ligand flexibility using different methods such systematic methods, stochastic methods and deterministic search ${ }^{[18]}$. The size and complexity of proteins makes it difficult to fully account for their mobility during a docking process and, therefore, its treatment is usually restricted to selected residues. Different approaches such as soft docking, side chain flexibility, combined protein grid, united description of the receptor are used in docking studies to incorporate protein flexibility.

MD simulations could possibly be a useful tool for receptor flexibility. Protein structures won't exist in single conformation and it forms different conformations at different energy states ${ }^{[19]}$. Different conformations won't be equally able to bind productively with a given ligand. Some conformations will accommodate the ligand molecule within the binding site, while others will be less likely because of loops that block the access to the binding site ${ }^{[20]}$. A group of receptor conformations is expected to provide a better representation of the system. Docking of small molecule against many conformations of receptor structures increases the chances of finding a receptor in the right conformational state to accommodate a particular ligand. Though, it also reduces the selectivity of the docking process, as a wider variety of ligands will be able to fit in this more relaxed representation of the protein. It is important, therefore, to use accurate scoring functions during the 
final screening process to maximize selection of the most active ligands. We conclude that the application of MD before the docking process offers a suitable approach to explore the conformational space of the protein receptor.

\section{Refinement of Docked Complexes}

A two-step protocol has been considered as the suitable approach to address the docking problem. At first scoring functions are used to virtual screen large number of small molecules against a receptor and reduce their size. It is then followed by the application of MD simulation methods to refine the protein ligand complexes and predict their energy of binding ${ }^{[21,22]}$. MD simulations consider the flexibility of both ligand and the receptor structures and proved to be a useful technique for structural refinement of docked complexes. The evolution of the complexes over the simulation time course is an indication of their stability and reliability. Incorrect docked complexes are likely to produce unstable trajectories, leading to the disruption of the complex; while realistic complexes will show stable behavior. In addition, MD simulations also consider explicit solvent molecules and their interactions in the simulations. Several studies have been reported by using MD simulation for refinement of docked complexes $^{[23,24]}$.

\section{Free Energy Calculations}

After successful refinement of docked complexes, it is necessary to estimate the absolute binding free energy. It can be achieved by the application of different MD-based calculations on the final complexes. Thermodynamic integration (TI) and free energy perturbation (FEP) are among the most rigorous methods currently available for the calculation of free energies. Though they are accurate in estimating free energies, they are computationally expensive. Recently developed approaches that provide relatively good energy values at a moderate cost include MD-based methods such as the linear interaction energy (LIE) method and the so-called MM-PBSA method. Several studies have been reported for the calculation of free energies $^{[25,26,27]}$.

\section{Conclusion}

In this short review, the combination of molecular dynamics and molecular docking methodologies was reviewed. The application of different strategies such as importance of protein flexibility, refinement of docked complexes using molecular dynamics and the application of free energy calculations to calculate binding energies was reviewed. Considering these strategies could be helpful in the process of drug discovery.

\section{References}

[1] D. Brown and G. Superti-Furga, "Rediscovering the sweet spot in drug discovery", Drug. Discov. Today., Vol. 8, pp. 1067-1077, 2003.

[2] J. Drews, "Stategic trends in the drug industry", Drug. Discov. Today., Vol. 8, pp. 411-420, 2003.

[3] R. Lahana, "How many leads from HTS?", Drug. Discov. Today. Vol. 4, pp. 447-448, 1999.

[4] H. Jhoti and A.R. Leach, "Structure-based drug discovery", Dordrecht, Springer, 2007.

[5] W. L. Jorgensen, "The many roles of computation in drug discovery", Science, Vol. 303, pp. 18131818, 2004.

[6] H. J. Boehm, M. Boehringer, D. Bur, H. Gmuender, W. Huber, W. Klaus, D. Kostrewa, H. Kuehne, T. Luebbers, N. Meunier-Keller, and F. Mueller, "Novel inhibitors of DNA gyrase: 3D structure based biased needle screening, hit validation by biophysical methods, and 3D guided optimization. A promising alternative to random screening", J. Med. Chem., Vol. 43, pp. 2664-2674, 2003.

[7] A. V. Filikov, V. Mohan, T. A. Vickers, R. H. Griffey, P. D. Cook, R. A. Abagyan, and T. L. James, "Identification of ligands for RNA targets via structure-based virtual screening: HIV-1 TAR", J. Comput. Aided. Mol. Des., Vol. 14, pp. 593-610, 2000.

[8] Y. Iwata, M. Arisawa, R. Hamada, Y. Kita, M. Y. Mizutani, N. Tomioka, A. Itai, and S. Miyamoto, "Discovery of novel aldose reductase inhibitors using a protein structure-based approach: 3Ddatabase search followed by design and synthesis", J. Med. Chem., Vol. 44, pp. 1718-1728, 2001.

[9] G. Kothandan, T. Madhavan, C. G. Gadhe, and S. J. Cho, "Pseudoreceptor: Concept and an Overview", J. Chosun Natural Sci., Vol. 5, pp. 216-219, 2012.

[10] G. Kothandan and S. J. Cho, "Prediction of Binding Free Energy Calculation Using Molecular Mechan- 
ics/Poisson-Boltzmann Surface Area (MM-PBSA) Method in Drug Discovery: A Short Review", J. Chosun Natural Sci., Vol. 2, pp. 55-58, 2009.

[11] S. J. Cho, "Search space reduction techniques in small molecular docking." J. Chosun Natural Sci., Vol. 3, pp. 143-147, 2010.

[12] S. J. Cho, "Calculation and application of partial charges", J. Chosun Natural Sci., Vol. 3, pp. 226230, 2010.

[13] S. J. Cho, "Meaning and definition of partial charges", J. Chosun Natural Sci., Vol. 3, pp. 231236, 2010.

[14] M. D. Cummings, R. L. Des Jarlais, A. C. Gibbs, V. Mohan, and E.P. Jaeger, "Comparison of automated docking programs as virtual screening tools", J. Med. Chem., Vol. 48, pp. 962-976, 2005.

[15] J. Norberg and L. Nilsson, "Advances in biomolecular simulations: Methodology and recent applications", Quart. Rev. Biophys., Vol. 36, pp. 257-306, 2003.

[16] J. A. Erickson, M. Jalaie, D. H. Robertson, R. A. Lewis, and M. Vieth, "Lessons in molecular recognition: The effects of ligand and protein flexibility on molecular docking accuracy", J. Med. Chem., Vol. 47, pp. 45-55, 2004.

[17] Y. Sugit and Y. Okamoto, "Replica-exchange molecular dynamics method for protein folding", Chem. Phys. Lett., Vol. 314, pp. 141-151, 1999.

[18] N. Brooijmans and I.D. Kuntz, "Molecular recognition and docking algorithms", Annu. Rev. Biophys. Biomol. Struct., Vol. 32, pp. 335-373, 2003.

[19] S. Kumar, B. Ma, C. J. Tsai, N. Sinha, and R. Nussinov, "Folding and binding cascades: Dynamic landscapes and population shifts", Protein Sci., Vol. 9, pp. 10-19, 2000.
[20] B. Ma, S. Kumar, C. J. Tsai, and R. Nussinov, "Folding funnels and binding mechanisms", Protein. Eng., Vol. 12, pp. 713-720, 1999.

[21] D. Hoffmann, B. Kramer, T. Washio, T. Steinmetzer, M. Rarey, and T. Lengauer, "Two-stage method for proteinligand docking", J. Med. Chem., Vol. 42, pp. 4422-4433, 1999.

[22] J. Wang, P. A. Kollman, and I. D. Kuntz, "Flexible ligand docking: A multistep strategy approach', Proteins, Vol. 36, pp. 1-19, 1999.

[23] H. Park, M. S. Yeom, and S. Lee, "Loop flexibility and solvent dynamics as determinants for the selective inhibition of cyclin-dependent kinase 4 : Comparative molecular dynamics simulation studies of CDK2 and CDK4", Chembiochem., Vol. 5, pp. 1662-1672, 2004.

[24] A. Cavalli, G. Bottegoni, C. Raco, M. de Vivo, and M. Recanatini, "A computational study of the binding of propidium to the peripheral anionic site of human acetylcholinesterase", J. Med. Chem., Vol. 47, pp. 3991-3999, 2004.

[25] H. Park and S. Lee, "Homologymodeling, force field design, and free energy simulation studies to optimize the activities of histone deacetylase inhibitors", J. Comput. Aided Mol. Des., Vol. 18, pp. 375-388, 2004.

[26] H. Park and M. S. Lee, "Free energy perturbation approach to the critical assessment of selective cyclooxygenase-2 inhibitors", J. Comput. Aided Mol. Des., Vol. 19, pp. 17-31, 2005.

[27] V. B. Luzhkov, F. Osterberg, and J. Aqvist, "Structure-activity relationship for extracellular block of Kpchannels by tetraalkylammonium ions", FEBS Lett., Vol. 554, pp. 159-164, 2003. 